\title{
Abnormal Expression of miRNAs in RCC
}

\author{
Bixuan Wang \\ College of Life Science, Sichuan University, Chengdu, PR China \\ 2017141241033@stu.scu.edu.cn
}

Keywords: renal cell carcinoma, miRNAs, biomarkers.

\begin{abstract}
Renal cell carcinoma (RCC) is one of the most common malignant tumors of urinary system, accounting for about 3\% of human malignant tumors, which is the tenth most common cancer in the world. However, there is a lack of accurate biomarkers for the diagnosis of RCC, and the mechanism of the occurrence and development of RCC is not clear. In recent years, many studies have focused on the abnormal expression of miRNAs in RCC. Studying the abnormal expression of miRNAs in RCC is helpful to understand the mechanism of occurrence and development of RCC, and to provide new ideas for the development of potential biomarkers and therapeutic targets of RCC.
\end{abstract}

\section{Introduction}

Renal cell carcinoma (RCC) is one of the most common malignant tumors of urinary system, accounting for about $3 \%$ of human malignant tumors, which is the tenth most common cancer in the world. In RCC, renal clear cell carcinoma (ccRCC) accounts for about 70\%, which is the third common urinary cancer after prostate cancer and bladder cancer, but its mortality is the highest[1]. Although more than $50 \%$ of stage I patients can be cured by simple or radical resection, the overall survival rate of stage IV patients is still very low. In addition, about $20 \%$ - 30\% of RCC patients have metastasis at the time of diagnosis, and $20 \%$ - $40 \%$ of RCC patients have metastasis after nephrectomy[2]. Therefore, the early diagnosis of RCC is the premise of effective treatment. At present, CT and histopathological analysis of biopsy are the gold standards for clinical diagnosis. In view of the high radiation dose of CT scan and the presence of more traumatic complications in pathological detection, new minimally invasive or non-invasive diagnostic tools are more beneficial to patients and clinicians. However, there is a lack of accurate biomarkers for the diagnosis of RCC, and the mechanism of the occurrence and development of RCC is not clear. Therefore, it is of great clinical significance to develop new biomarkers and study their mechanism of action to improve the early diagnosis and prognosis of RCC.

MicroRNAs (miRNAs, miR) are small, evolutionarily conserved non coding RNAs, with a length of 18-24 nucleotides, which can bind to the 3 ' untranslated region (3' - UTR) of the target mRNA and inhibit its translation or induce its degradation, thus negatively regulate the expression of the target gene. MiRNAs are important regulators of cell proliferation, differentiation, development and death. They are involved in many biological and pathological processes such as proliferation, apoptosis, metastasis and invasion of RCC. More and more evidences show that the abnormal expression of miRNAs is a sign of the occurrence and development of cancer. MiRNAs can play a role as oncomiRs by inhibiting tumor suppressor genes, and can act as tumor suppressor genes by negatively regulating oncogenes[3]. In recent years, many studies have focused on the abnormal expression of miRNAs in RCC. Studying the abnormal expression of miRNAs in RCC is helpful to understand the mechanism of occurrence and development of RCC, and to provide new ideas for the development of potential biomarkers and therapeutic targets of RCC. In this review, I will summarize the research progress of abnormal expression of miRNAs and its mechanism in RCC in recent years. 


\section{Upregulated miRNAs in RCC}

Some upregulated miRNAs in RCC may play the role of oncogenes by inhibiting tumor suppressors, and then affect the proliferation, apoptosis, metastasis and invasion of RCC. Many evidences show that miR-126 is abnormally expressed in many types of cancers including esophageal carcinoma, lung cancer, urothelium carcinoma, breast cancer, cutaneous squamous cell carcinoma and RCC. MiR-126 regulates genes in the vascular endothelial growth factor and phosphatidylinositol 3-kinase pathways, so it plays an important role in angiogenesis and cell cycle regulation. Carlsson et al. used RT-qPCR to detect the expression of miR-126, miR-21 and miR-10b in renal tissue of 116 patients with ccRCC. It was found that the expression of miR-126 was different in malignant and benign tissues, and the low expression of miR-126 was related to shorter time to recurrence, which had the greatest prognostic potential of the three miRNAs and could be used as a prognostic marker for ccRCC patients[4].

Many studies have shown that miR-210 is overexpressed in patients with ccRCC. The deregulation of miR-210 expression is closely related to the pathogenesis of ccRCC. Wang et al. showed that the expression of miR-210 in tumor tissue was upregulated compared with normal tissue, and the level of miR-210 in serum of ccRCC patients was higher than that of healthy control group, suggesting that miR-210 might be a new noninvasive biomarker for the detection and prognosis of ccRCC. They found that the overexpression of miR-210 reduced the expression of vacuole membrane protein 1 (VMP1) and promoted cell metastasis and invasion. They further speculated that VMP1 might be the target of miR-210[5]. Other studies confirmed that miR-210-3p was upregulated in tumor tissues and urine samples of patients with ccRCC, and the level of miR-210-3p in urine was significantly reduced. This shows that miR-210-3p can be used as a biomarker in the monitoring of ccRCC tumor progression, which is not only helpful for diagnosis, but also for the evaluation of complete resection and disease-free survival rate of patients with ccRCC during follow-up[6].

MiR-21 has been confirmed to be upregulated in a variety of cancers including RCC, and it is the most stably expressed miRNA, which is expected to become a biomarker and therapeutic target of ccRCC. The early event of renal cancer is the inactivation of tumor suppressor von Hippel Lindau (VHL), which is the direct target of miR-21. Some studies have found that miR-21 expression in ccRCC tumor tissue samples is upregulated compared with adjacent non tumor tissue samples. Cao et al. have confirmed that miR-21 is an oncomiR of ccRCC, which can promote the formation of highly tumorigenic cancer spheres in renal cancer[7].

MiR-625-3p has been found to play an inducible or inhibitory role in bladder cancer, esophageal squamous cell carcinoma and breast cancer. Zhao et al. confirmed that the expression of miR-625-3p was upregulated in ccRCC compared with normal tissues, which was related to poor prognosis of patients. The expression of miR-625-3p was downregulated in the serum of patients with ccRCC, which can distinguish between patients with ccRCC and normal people. In addition, miR-625-3p promoted the metastasis and invasion of ccRCC in vitro, but inhibited the apoptosis of ccRCC cells. Bioinformatics analysis showed that the target of miR-625-3p was related to the poor prognosis of patients with ccRCC[8]. Therefore, miR-625-3p is expected to be a biomarker for diagnosis and prognosis of ccRCC and a potential therapeutic target.

MiR-15a is downregulated in prostate cancer, nasopharyngeal cancer and breast cancer. As a tumor suppressor, miR-15a can promote cell apoptosis and inhibit cell proliferation by targeting a variety of oncogenes, such as Bcl-2, MCL1, CCND1 and Wnt3a[9]. Mytsyk et al. studied the expression of miR-15a in the urine of RCC patients, and found that compared with benign renal tumor patients and healthy control group, the expression of miR-15a in RCC patients before operation was significantly upregulated, and that in RCC patients after operation was significantly downregulated, suggesting that the expression of miR-15a in the urine can be used as a biomarker for RCC diagnosis. Their subsequent study confirmed that the expression of miR-15a in RCC patients' tumor tissue was significantly upregulated compared with the healthy control group, and the over expression of miR-15a was closely related to the poor prognosis of RCC, indicating that the expression of miR-15a in the tissue can be used as a molecular marker of RCC prognosis [10]. 
MiR-935 was found to be upregulated in pancreatic cancer, liver cancer and gastric cancer, which plays a role in promoting the proliferation, metastasis and invasion of cancer cells. Liu et al. found that miR-935 was upregulated in ccRCC cells and cancer tissues while iron responsive element binding protein 2 (IREB2) was downregulated in ccRCC cancer tissues, and IREB2 with high expression had longer overall survival (OS) and disease-free survival (DFS), which revealed that miR-935 promoted the proliferation, metastasis and invasion of RCC cells through its direct target IREB2 carcinogenic effect[11]. This study shows that miR-935, as an oncomiR, may be a potential biomarker for the diagnosis of ccRCC, thus providing a new strategy for the diagnosis and treatment of cCRCC.

\section{Downregulated miRNAs in RCC}

The expression of tumor suppressor miRNAs targeting oncogene in RCC are generally downregulated, which leads to the activation of oncogene pathway, and then affects the proliferation, apoptosis, metastasis and invasion of RCC.

Abnormal expression of miR-363 was found in gastric cancer, prostate cancer, lung cancer and colorectal cancer. MiR-363 is also one of the most significantly downregulated miRNAs in ccRCC. Xie et al. found that the expression of miR-363 was significantly downregulated in ccRCC tissues, and was associated with poor prognosis. The mechanism is that miR-363 can inhibit the proliferation, metastasis and invasion of ccRCC cells by downregulating the expression of the target sphingosine-1-phosphote receptor 1 (S1PR1) and further affecting ERK and other downstream genes, leading to the inhibition of the growth of tumors. Therefore, miR-363/S1PR1/ERK pathway is expected to be a new target of ccRCC treatment[12].

MiR-125 family members play a regulatory role in breast cancer, ovarian cancer, bladder cancer, liver cancer and leukemia. MiR-125a/b can inhibit the expression of vascular endothelial growth factor (VEGF), thereby inhibiting the growth and angiogenesis of various tumors[13]. Hou et al. found that the expression of miR-125a-3p was significantly downregulated in RCC patients, and the low expression of miR-125a-3p was associated with poor prognosis in these patients. The tumor suppressor PinX1 inhibits the angiogenesis of RCC through miR-125a-3p/VEGF signal pathway[14]. Therefore, miR-125a-3p may be a candidate prognostic marker and a new therapeutic target for RCC.

A large number of evidences show that miR-30a-3p plays an important role in the inhibition of breast cancer, liver cancer and lung adenocarcinoma. Some studies have confirmed that the expression level of miR-30a-3p in RCC tissue and cell line is significantly lower than that in normal tissue and HK-2 cell line, and found that autophagy related protein 12 (ATG12), as the downstream target gene of miR-30a-3p, is highly expressed in RCC cells, which is negatively related to the prognosis of RCC. MiR-30a-3p can downregulate the invasion and metastasis of RCC cells by targeting ATG12[15]. Therefore, miR-30a-3p has the potential to be a new prognostic marker of RCC.

Mir-766 plays an important role in the induction and inhibition of human cancers such as cutaneous squamous cell carcinoma, lung adenocarcinoma and acute promyelocytic leukemia. MicroRNA expression analysis of the whole genome showed that miR-766-3p was one of the most downregulated miRNAs in RCC[16]. Chen et al. confirmed that the methylation of miR-766-3p promoter in RCC was higher than that in non-tumor tissues, and the downregulation of miR-766-3p was significantly related to the clinical stage and poor prognosis of RCC patients. They revealed that spreading factor 2 (SF2) is the target of miR-766-3p, which acts as a tumor suppressor of renal cancer cells and inhibits the proliferation of renal cancer cells by regulating the SF2/p-Akt/p-ERK signaling pathway[17].

Some studies have found that the expression of miR-200a may play an important role in the inhibition of human cancer, and in the epithelial-mesenchymal transition (EMT). It has been reported that the expression level of miR-200a in RCC samples is significantly lower, and it has inhibitory effect on RCC[18]. Fu et al. confirmed that the expression of miR-200a in RCC samples and RCC 
cell lines was significantly downregulated. Upregulation of miR-200a expression can directly inhibit cell proliferation and induce cell apoptosis as well as cell cycle arrest. They revealed that miR-200a can directly target sirtuin 1 (SIRT1) to play an anti-cancer role in RCC tissues, providing new ideas for developing RCC treatment strategies by targeting miR-200a or SIRT1[19].

Previous studies have shown that miR-372 is involved in malignant tumors such as brain cancer, gastric cancer, colon cancer and glioma, and found that miR-372 is upregulated while playing a carcinogenic role. However, Huang et al. found that miR-372 was downregulated in RCC cell lines and tissues, and miR-372 inhibited the proliferation and invasion of RCC cells through its direct target insulin-like growth factor-2 mRNA binding protein 1 (IGF2BP1), revealing that miR-372 may play an anti-cancer role in the development of RCC, providing new insight for the treatment of RCC[20].

\section{Conclusion}

At present, the main treatment of RCC is surgery. But after surgery, nearly half of RCC patients still have relapse and metastasis, and chemotherapy or radiotherapy is not effective. Therefore, it is necessary to find effective methods of early diagnosis and treatment to inhibit the malignant progression of RCC. In this respect, the search for useful tumor biomarkers is not only a method of early diagnosis, but also a new way to treat RCC. However, there is no biomarker for early diagnosis or prognosis in the routine clinical practice of RCC, and the mechanism of RCC occurrence and development is not clear. Although some progress has been made in the search for biomarkers, most of which are based on tissue or cell level and invasive surgery or puncture is inevitable. But from a clinical point of view, it is very significant to screen biomarkers from serum or urine and non-invasive and effective biomarkers are important directions that need to be explored. The research of abnormally expressed miRNAs extends the research of RCC biomarkers. Abnormal expression of miRNAs is common in human cancer. There are many studies on the abnormal expression of miRNAs in various cancers including RCC. At present, many abnormally expressed miRNAs have been found in RCC, some miRNAs are significantly upregulated, while others are significantly downregulated. More and more evidences show that these miRNAs, as oncomiRs or tumor suppressors, widely regulate the biological process of cells, and are closely related to the occurrence and development of RCC. The identification of these miRNAs, their target genes and the key pathways they participating in helps to deepen the understanding of the molecular mechanisms of the occurrence and development of RCC, and is essential for developing new cancer prevention strategies. These miRNAs are expected to become new biomarkers for the diagnosis and prognosis of RCC, or as potential therapeutic targets, having broad application prospects in the diagnosis, prognosis and treatment of RCC. However, the clinical application of these new biomarkers needs further research and verification in more patients.

\section{References}

[1] Novick AC. Kidney cancer: past, present, and future. Urol Oncol. 2007;25(3):188-195. doi:10.1016/j.urolonc.2007.03.006

[2] Nelson KM, Weiss GJ. MicroRNAs and cancer: past, present, and potential future. Mol Cancer Ther. 2008;7(12):3655-3660. doi:10.1158/1535-7163.MCT-08-0586

[3] Szabó Z, Szegedi K, Gombos K, et al. Expression of miRNA-21 and miRNA-221 in clear cell renal cell carcinoma (ccRCC) and their possible role in the development of ccRCC. Urol Oncol. 2016;34(12):533.e21-533.e27. doi:10.1016/j.urolonc.2016.06.011

[4] Carlsson J, Christiansen J, Davidsson S, Giunchi F, Fiorentino M, Sundqvist P. The potential role of miR-126, miR-21 and miR-10b as prognostic biomarkers in renal cell carcinoma. Oncol Lett. 2019;17(5):4566-4574. doi:10.3892/ol.2019.10142 
[5] Wang X, Wang T, Chen C, et al. Serum exosomal miR-210 as a potential biomarker for clear cell renal cell carcinoma [published online ahead of print, 2018 Oct 10]. J Cell Biochem. 2018;10.1002/jcb.27347. doi:10.1002/jcb.27347

[6] Petrozza V, Costantini M, Tito C, et al. Emerging role of secreted miR-210-3p as potential biomarker for clear cell Renal Cell Carcinoma metastasis. Cancer Biomark. 2020;27(2):181-188. doi:10.3233/CBM-190242

[7] Cao J, Liu J, Xu R, Zhu X, Liu L, Zhao X. MicroRNA-21 stimulates epithelial-to-mesenchymal transition and tumorigenesis in clear cell renal cells. Mol Med Rep. 2016;13(1):75-82. doi:10.3892/mmr.2015.4568

[8] Zhao L, Liu K, Pan X, et al. miR-625-3p promotes metastasis and invasion and reduces apoptosis of clear cell renal cell carcinoma. Am J Transl Res. 2019;11(10):6475-6486. Published 2019 Oct 15.

[9] Zhu K, He Y, Xia C, et al. MicroRNA-15a Inhibits Proliferation and Induces Apoptosis in CNE1 Nasopharyngeal Carcinoma Cells. Oncol Res. 2016;24(3):145-151. doi:10.3727/096504016X14611963142290

[10] Mytsyk Y, Borys Y, Tumanovska L, et al. MicroRNA-15a tissue expression is a prognostic marker for survival in patients with clear cell renal cell carcinoma. Clin Exp Med. 2019;19(4):515-524. doi:10.1007/s10238-019-00574-7

[11] Liu F, Chen Y, Chen B, Liu C, Xing J. MiR-935 Promotes Clear Cell Renal Cell Carcinoma metastasis and Invasion by Targeting IREB2. Cancer Manag Res. 2019;11:10891-10900. Published 2019 Dec 30. doi:10.2147/CMAR.S232380

[12] Xie Y, Chen L, Gao Y, et al. miR-363 suppresses the proliferation, metastasis and invasion of clear cell renal cell carcinoma by downregulating S1PR1. Cancer Cell Int. 2020;20:227. Published 2020 Jun 10. doi:10.1186/s12935-020-01313-9

[13] Bi Q, Tang S, Xia L, et al. Ectopic expression of MiR-125a inhibits the proliferation and metastasis of hepatocellular carcinoma by targeting MMP11 and VEGF. PLoS One. 2012;7(6):e40169. doi:10.1371/journal.pone.0040169

[14] Hou $\mathrm{P}$, Li $\mathrm{H}$, Yong $\mathrm{H}$, et al. PinX1 represses renal cancer angiogenesis via the mir-125a-3p/VEGF signaling pathway. Angiogenesis. 2019;22(4):507-519. doi:10.1007/s10456-019-09675-z

[15] Chen Y, Zhou J, Wu X, et al. miR-30a-3p inhibits renal cancer cell invasion and metastasis through targeting ATG12. Transl Androl Urol. 2020;9(2):646-653. doi:10.21037/tau.2019.12.10

[16] Osanto S, Qin Y, Buermans HP, et al. Genome-wide microRNA expression analysis of clear cell renal cell carcinoma by next generation deep sequencing. PLoS One. 2012;7(6):e38298. doi:10.1371/journal.pone.0038298

[17] Chen C, Xue S, Zhang J, et al. DNA-methylation-mediated repression of miR-766-3p promotes cell proliferation via targeting SF2 expression in renal cell carcinoma. Int J Cancer. 2017;141(9):1867-1878. doi:10.1002/ijc.30853

[18] Wang X, Jiang F, Song H, Li X, Xian J, Gu X. MicroRNA-200a-3p suppresses tumor proliferation and induces apoptosis by targeting SPAG9 in renal cell carcinoma. Biochem Biophys Res Commun. 2016;470(3):620-626. doi:10.1016/j.bbrc.2016.01.095

[19] Fu H, Song W, Chen X, et al. MiRNA-200a induce cell apoptosis in renal cell carcinoma by directly targeting SIRT1. Mol Cell Biochem. 2018;437(1-2):143-152. doi:10.1007/s11010-017-3102-1 
[20] Huang X, Huang M, Kong L, Li Y. miR-372 suppresses tumour proliferation and invasion by targeting IGF2BP1 in renal cell carcinoma. Cell Prolif. 2015;48(5):593-599. doi:10.1111/cpr.12207 